\title{
Reduced drug incorporation into DNA and antiapoptosis as the crucial mechanisms of resistance in a novel nelarabine-resistant cell line
}

\author{
Takahiro Yamauchi, Kanako Uzui, Rie Nishi, Hiroko Shigemi and Takanori Ueda
}

\begin{abstract}
Background: Nine-beta-D-arabinofuranosylguanine (ara-G), an active metabolite of nelarabine, enters leukemic cells through human Equilibrative Nucleoside Transporter 1, and is then phosphorylated to an intracellular active metabolite ara-G triphosphate (ara-GTP) by both cytosolic deoxycytidine kinase and mitochondrial deoxyguanosine kinase. Ara-GTP is subsequently incorporated into DNA, thereby inhibiting DNA synthesis.

Methods: In the present study, we developed a novel ara-G-resistant variant (CEM/ara-G) of human T-lymphoblastic leukemia cell line CCRF-CEM, and elucidated its mechanism of ara-G resistance. The cytotoxicity was measured by using the growth inhibition assay and the induction of apoptosis. Intracellular triphosphate concentrations were quantitated by using HPLC. DNA synthesis was evaluated by the incorporation of tritiated thymidine into DNA. Protein expression levels were determined by using Western blotting.

Results: CEM/ara-G cells were 70-fold more ara-G-resistant than were CEM cells. CEM/ara-G cells were also refractory to ara-G-mediated apoptosis. The transcript level of human Equilibrative Nucleoside Transporter 1 was lowered, and the protein levels of deoxycytidine kinase and deoxyguanosine kinase were decreased in CEM/ara-G cells. The subsequent production of intracellular ara-GTP $\left(21.3 \mathrm{pmol} / 10^{7}\right.$ cells) was one-fourth that of CEM cells $\left(83.9 \mathrm{pmol} / 10^{7}\right.$ cells) after incubation for $6 \mathrm{~h}$ with $10 \mu \mathrm{M}$ ara-G. Upon ara-G treatment, ara-G incorporation into nuclear and mitochondrial DNA resulted in the inhibition of DNA synthesis of both fractions in CEM cells. However, DNA synthesis was not inhibited in CEM/ara-G cells due to reduced ara-G incorporation into DNA. Mitochondrial DNA-depleted CEM cells, which were generated by treating CEM cells with ethidium bromide, were as sensitive to ara-G as CEM cells. Anti-apoptotic BCl-xL was increased and pro-apoptotic Bax and Bad were reduced in CEM/ara-G cells.
\end{abstract}

Conclusions: An ara-G-resistant CEM variant was successfully established. The mechanisms of resistance included reduced drug incorporation into nuclear DNA and antiapoptosis.

Keywords: Ara-G, Ara-GTP, Nelarabine, Resistance, T-ALL

\section{Background}

Nucleoside analogs belong to one of the most clinically useful and frequently used classes of agents for the treatment of hematological malignancies [1-6]. Nelarabine, 2-amino-9- $\beta$-D-arabinofuranosyl-6-methoxy-9H-purine, is a relatively new anticancer agent that targets $\mathrm{T}$-cell malignancies, including T-cell acute lymphoblastic leukemia and T-cell lymphoblastic lymphoma [4-6]. The Cancer and Leukemia Group B conducted a phase 2 study of

\footnotetext{
*Correspondence: tyamauch@u-fukui.ac.jp

Department of Hematology and Oncology, Faculty of Medical Sciences, University of Fukui, 23-3, Shimoaizuki, Matsuoka, Fukui 910-1193, Japan
}

nelarabine for adult patients with relapsed or refractory T-cell leukemia/lymphoma [7]. Treatment with nelarabine resulted in a $41 \%$ response rate and a $31 \%$ complete remission rate. Although this clinical outcome is promising, nelarabine therapy should be further optimized by an improved understanding of its mechanism of action and by overcoming drug resistance.

Upon intravenous administration, nelarabine is demethylated to the active compound 9- $\beta$-D-arabinofuranosylguanine (ara-G) by adenosine deaminase in the plasma [4,8-11]. Ara-G is transported into leukemic cells mainly via nitrobenzylthioinosine-sensitive nucleoside membrane 
transporter human Equilibrative Nucleoside Transporter 1 (hENT1) [12]. Ara-G is then phosphorylated to ara-G monophosphate by cytoplasmic deoxycytidine kinase (dCK) and mitochondrial deoxyguanosine kinase (dGK) [9]. This phosphorylation is the rate-limiting step of the intracellular activation of nelarabine. Ara-G nucleotide is partly dephosphorylated by cytosolic $5^{\prime}$-nucleotidase II (cN-II). Ara-G monophosphate is then phosphorylated to ara-G diphosphate and eventually to ara-G triphosphate (ara-GTP). Ara-GTP is an intracellular active metabolite, which is subsequently incorporated into both nuclear and mitochondrial DNA, thereby terminating DNA elongation. Thus, incorporation of the drug into DNA is critical for its cytotoxicity [8-10].

Nelarabine resistance is a major obstacle to improving response rates, and overcoming this drug resistance would provide new strategies for optimal nelarabine administration. In the present study, we established a novel ara-G-resistant subclone of the human T-cell lymphoblastic leukemia cell line, CCRF-CEM. Factors involved in the intracellular activation of ara-G that might be closely related to ara-G resistance [8-12], including hENT1, dCK, dGK, cN-II, and drug incorporation into DNA, were extensively investigated. Because ara-G is phosphorylated by cytoplasmic dCK and mitochondrial dGK, the contribution of both nuclear and mitochondrial DNA damage was evaluated. Moreover, because the induction of apoptosis is the final output of mechanism of ara-G cytotoxicity, the levels of apoptosisrelated proteins were determined.

\section{Methods}

\section{Reagents}

Ara-G was purchased from R.I. Chemicals (Orange, CA, USA) and dissolved in 100\% dimethyl sulfoxide. Standard ara-GTP was provided by GlaxoSmithKline, Japan (Tokyo, Japan). $\left[5-{ }^{3} \mathrm{H}\right]$ ara-G $(30 \mathrm{Ci} / \mathrm{mmol})$ was purchased from Moravek Biochemicals, Inc (Brea, CA, USA). Nine- $\beta-D-$ arabinofucanosyl-2-fluoroadenine (F-ara-A) and cytarabine (ara-C) were purchased from Sigma-Aldrich (St Louis, MO, USA).

\section{Cell culture and development of an ara-G-resistant subclone}

Human T-cell lymphoblastic leukemia CCRF-CEM cells were cultured in RPMI1640 media supplemented with $10 \%$ fetal calf serum. An ara-G-resistant variant, CEM/ ara-G, was established by serial incubation of the cells with ara-G, followed by limiting dilution for cloning. In brief, the parental CEM cells were maintained with escalating concentrations of ara-G. The initial concentration $(0.2 \mu \mathrm{M})$ was one tenth the concentration required to inhibit $50 \%$ growth of CEM cells $\left(\mathrm{IC}_{50}\right)$. The cultures were observed daily and allowed to grow. In subsequent passages, the concentration of ara-G was gradually increased. Passaging was repeated for 10 months. When the ara-G concentration in the culture media reached $20 \mu \mathrm{M}$, one cell line resistant to ara-G (CEM/ara-G) was cloned by the limiting dilution method [13].

\section{Drug treatment}

Both CEM and CEM/ara-G cells $\left(2 \times 10^{6}\right.$ cells $\left./ \mathrm{ml}, 10 \mathrm{ml}\right)$ were incubated at $37^{\circ} \mathrm{C}$ with various concentrations of radiolabeled or non-labeled ara-G for the time periods indicated. Cells were then washed twice with PBS and centrifuged $\left(500 \times \mathrm{g}, 5 \mathrm{~min}, 4^{\circ} \mathrm{C}\right)$ to collect the cell pellet.

\section{Proliferation assay}

Growth inhibition effects were determined by the sodium 3'-(1-[(phenylamino)-carbonyl-3,4-tetrazolium])-bis (4-methoxy-6-nitro) benzene sulfonic acid hydrate (XTT) assay according to the manufacturer's instructions (Roche, Indianapolis, IN, USA) with slight modifications [13].

Alternatively, the number of viable cells were quantitated as of the ATP present, which signals the presence of metabolically active cells, by using The CellTiter-Glo ${ }^{\circ}$ Luminescent Cell Viability Assay kit (Promega Corp., Madison, WI, USA). Briefly, the cell suspension having been treated were added to the reagent $(1: 1, \mathrm{v} / \mathrm{v})$. The sample was mixed for 2 min for cell lysis, and allowed to stand for $10 \mathrm{~min}$ to stabilize the luminescent signal. The luminescence intensity of the sample was measured thereafter. This method was applied to assess the viability of mitochondrial DNA-depleted $\rho^{0} \mathrm{CEM}$ cells.

\section{Measurement of analog triphosphate concentrations in leukemic cells}

Intracellular concentrations of ara-GTP, F-ara-A triphosphate (F-ara-ATP), and ara-C triphosphate (ara-CTP) were determined by using the HPLC assay method that we previously established $[13,14]$. Briefly, cells $\left(1 \times 10^{6}\right.$ cells $/ \mathrm{ml}$, $10 \mathrm{ml}$ ) were incubated for $6 \mathrm{~h}$ with $10 \mu \mathrm{M}$ ara-G, F-ara-A, or ara-C. The acid-soluble fraction, the nucleotide pool, was extracted from the cells by the addition of perchloric acid followed by neutralization. An aliquot of the sample was subjected to HPLC analysis. Chromatography was performed with the TSK gel DEAE-2 SW column (250 mm length $\times 4.6 \mathrm{~mm}$ inside diameter; Tosoh, Tokyo, Japan) and $0.06 \mathrm{M} \mathrm{Na}_{2} \mathrm{HPO}_{4}(\mathrm{pH} 6.9)-20 \%$ acetonitrile buffer at a constant flow rate of $0.7 \mathrm{ml} / \mathrm{min}$. Each analog triphosphate concentration was quantitated by its peak area and expressed as pmol/10 ${ }^{7}$ cells.

\section{Western blot analysis}

Protein levels of dCK, dGK, caspase-3, caspase-9, Bcl2, $\mathrm{Bcl}-\mathrm{xL}, \mathrm{Bax}, \mathrm{Bad}, \mathrm{Bid}, \mathrm{Bim}, \mathrm{AKT}$, and p-AKT were determined by using standard western blotting techniques [13]. Mouse monoclonal anti-dCK was developed in the 
Department of Pediatrics of Mie University School of Medicine [13]. Rabbit polyclonal anti-dGK antibody (Abgent, San Diego, CA, USA), rabbit polyclonal anticaspase-3 (Cell Signaling Technology, Beverly, MA, USA), rabbit polyclonal anti-caspase-9 (Cell Signaling Technology), rabbit polyclonal anti-Bcl-2 (Cell Signaling Technology), rabbit polyclonal anti-Bcl-xL (Cell Signaling Technology), rabbit polyclonal anti-Bax (Cell Signaling Technology), rabbit polyclonal anti-Bad (Cell Signaling Technology), rabbit polyclonal anti-Bid (Cell Signaling Technology), rabbit polyclonal anti-Bim (Cell Signaling Technology), rabbit polyclonal anti-AKT (Cell Signaling Technology), rabbit polyclonal anti-P-AKT (Santa Cruz Biotechnology, Inc. Dallas, TX, USA), and antiactin antibodies (Sigma-Aldrich) were used as primary antibodies [13].

\section{Determination of hENT1 and $\mathrm{cN}$-II transcripts}

To evaluate mRNA levels of hENT1 (accession: NM_ 001078177) and cN-II (accession: NM_012229), real-time RT-PCR was performed by using the ABI Prism 7900 sequence detection system (Applied Biosystems, Foster City, CA, USA) as previously described $[13,15]$. Primers for hENT1 and cN-II were purchased from Applied Biosystems. The relative quantification method was used. The expression level of hENT1 or cN-II was normalized using $\beta$-Actin as a house-keeping gene in each cell line. The final value was expressed as the ratio of the expression level of hENT1 or $\mathrm{cN}-\mathrm{II}$ of CEM/ara-G cells to that of CEM cells (the expression level of hENT1 or cN-II of CEM cells was set as 1 ).

\section{Calculation of ara-G incorporation into both nuclear and mitochondrial DNA}

Both nuclear and mitochondrial DNA fractions were isolated from cells after incubation with tritiated ara-G for the indicated time periods at $37^{\circ} \mathrm{C}$. For nuclear DNA isolation, the acid-insoluble fraction (obtained as described above) was used. To solubilize RNA, the acid-insoluble fraction was resuspended in $100 \mu \mathrm{l}$ of 0.4 $\mathrm{N} \mathrm{KOH}$ and kept at room temperature for $4 \mathrm{~h}$. The sample was then mixed with $100 \mu \mathrm{l}$ of $5 \%$ perchloric acid and $20 \mu \mathrm{l}$ of $4 \mathrm{~N} \mathrm{HCl}$, followed by centrifugation $\left(15,000 \times \mathrm{g}, 30 \mathrm{sec}, 4^{\circ} \mathrm{C}\right)$. After removal of the supernatant (RNA), the precipitate was mixed with $100 \mu \mathrm{l}$ of $5 \%$ perchloric acid and heated at $92^{\circ} \mathrm{C}$ for $20 \mathrm{~min}$ to solubilize DNA. After centrifugation $(15,000 \times \mathrm{g}, 30 \mathrm{sec}$, $4^{\circ} \mathrm{C}$ ), the supernatant was isolated as DNA, and the precipitate (protein) was discarded [16]. The mitochondrial fraction was extracted by using the Qproteome Mitochondria Isolation Kit (Qiagen, Valencia, CA, USA) according to the manufacturer's instructions. Radioactivity was determined in both fractions by using a liquid scintillation counter.

\section{Evaluation of nuclear and mitochondrial DNA synthesis}

The inhibition of DNA synthesis by ara-G was evaluated by assessing the incorporation of tritiated thymidine into DNA [17]. Cells $\left(2 \times 10^{6}\right.$ cells $)$ were pre-incubated with or without $10 \mu \mathrm{M}$ ara-G for $3 \mathrm{~h}$, followed by washing in fresh media and subsequent incubation with tritiated thymidine for $4 \mathrm{~h}$. The nuclear and mitochondrial DNA fractions were extracted as described above and evaluated for radioactivity by using a liquid scintillation counter.

\section{Quantitation of apoptotic cell death}

To evaluate cytotoxicity, apoptotic cell death was determined by staining for phosphatidylserine externalization by using annexin V (Roche Applied Science, Indianapolis, IN, USA) or for the sub-G1 cell cycle population by using propidium iodide (Beckman Coulter, Fullerton, CA, USA) and performing flow cytometry $72 \mathrm{~h}$ after treatment [18]. To confirm the induction of mitochondrial apoptosis, the cleavage of caspase- 3 and caspase- 9 was detected by western blotting as described above.

\section{Derivation of mitochondrial DNA-depleted cells ( $\rho^{0} \mathrm{CEM}$ cells)}

CEM cells were cultured in the presence of $100 \mathrm{ng} / \mathrm{ml}$ ethidium bromide to inhibit mitochondrial DNA replication for more than 20 generations (almost 1 month) [19]. $\rho^{0}$ cells were derived and maintained in the presence of $50 \mathrm{mg} / \mathrm{ml}$ uridine. The total cellular enzyme activity of cytochrome c oxidase, subunits of which are encoded by mitochondrial DNA, was tested by using the Mitochondrial Activity Assay Kit (BioChain, Institute, Inc., Hayward, CA, USA) according to the manufacturer's instructions.

\section{Statistical analyses}

All statistical analyses were performed with Microsoft Excel 2007 (Microsoft Corporation, Redmond, WA, USA). All graphs were generated using GraphPad Prism (version 5.0; GraphPad Software, San Diego, CA, USA).

\section{Results}

\section{Establishment of ara-G-resistant CEM (CEM/ara-G) cells}

The XTT proliferation assay demonstrated that CEM/ ara-G cells were 70 -fold more resistant to ara-G than CEM cells (Figure 1a, Table 1). Because growth rates for both cell lines were similar (Figure 1b) with a doubling time of $22.0 \mathrm{~h}$ for CEM cells and $21.4 \mathrm{~h}$ for CEM/ara-G cells, the resistance to this S-phase-specific drug was not attributable to cycling speed. The intracellular ara-GTP production (21.3 pmol $/ 10^{7}$ cells) was reduced by $1 / 4$ in CEM/ara-G cells compared with that (83.9 pmol/10 ${ }^{7}$ cells) in CEM cells (Figure 1c). CEM/ ara-G cells were also resistant to ara-G-induced apoptosis 


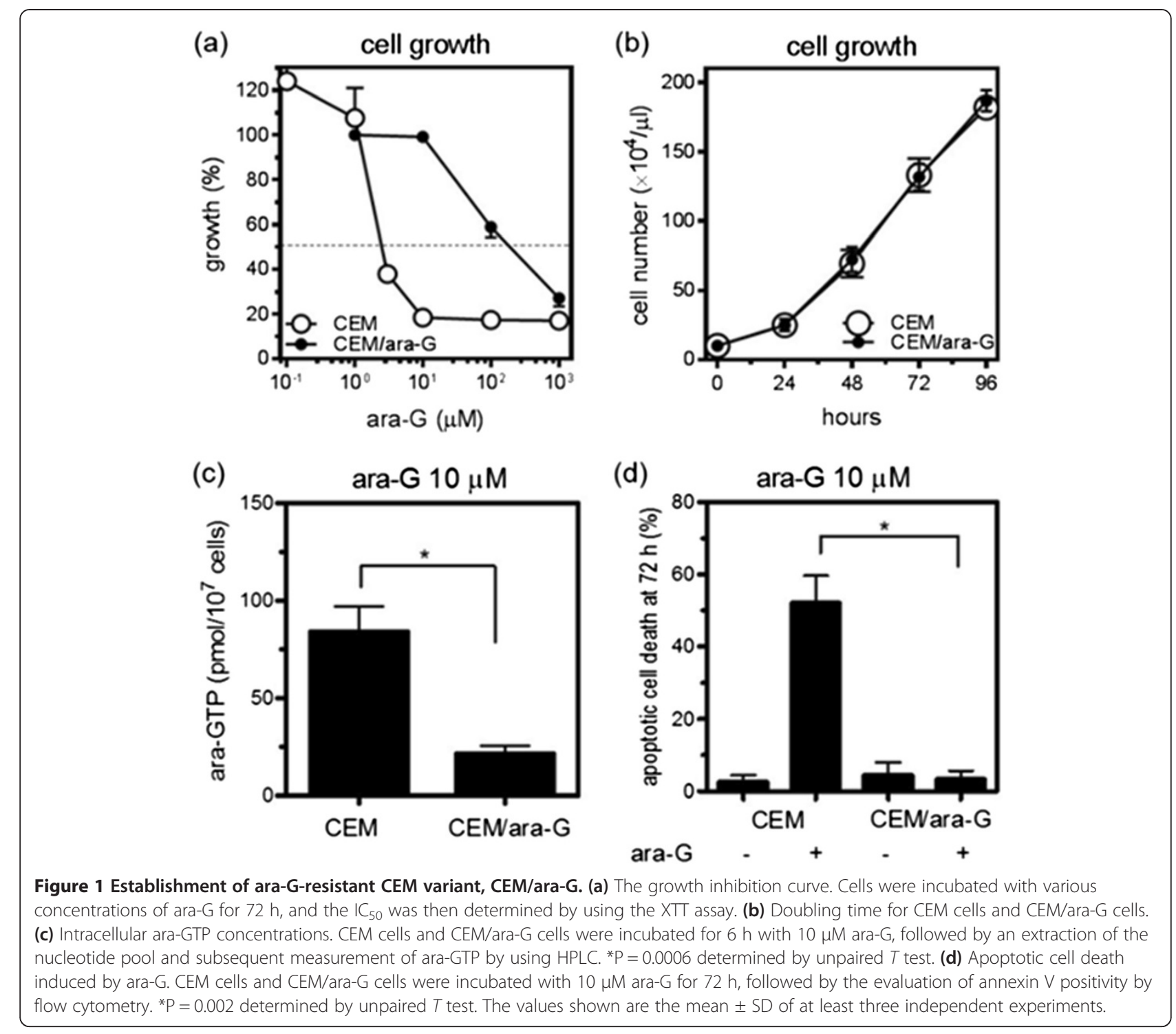

(Figure 1d). Cleavage of caspase 3 and caspase 9 was demonstrated in CEM cells treated with ara-G, suggesting that mitochondria-mediated apoptosis was induced by ara-G (Figure 2). In contrast, caspase cleavage was not induced in CEM/ara-G cells treated with $100 \mu \mathrm{M}$

Table 1 Drug sensitivities of CEM and CEM/ara-G cells

\begin{tabular}{lccc}
\hline Drug & \multicolumn{3}{c}{$\mathbf{I C}_{\mathbf{5 0}}(\boldsymbol{\mu} \mathbf{M})$} \\
\cline { 2 - 4 } & $\mathbf{C E M}$ & $\mathbf{C E M} /$ ara-G & $\mathbf{R R}$ \\
\hline Ara-G & 2.6 & 180 & $(70)$ \\
F-ara-A & 0.10 & 4.80 & $(48)$ \\
Ara-C & 0.15 & 0.75 & $(5)$
\end{tabular}

CEM and CEM/ara-G cells were incubated for $72 \mathrm{~h}$ with various concentrations of ara-G, ara-C, or F-ara-A. The $\mathrm{IC}_{50}$ was then determined by using the XTT assay. The number in the parenthesis is the relative resistance (RR), which was obtained by dividing the $\mathrm{IC}_{50}$ value of CEM/ara-G cells by that of CEM cells.
ara-G (Figure 2). Thus, the ara-G-resistant CEM variant, CEM/ara-G, was successfully established, which yielded a small amount of ara-GTP and was consequently more resistant to ara-G-induced growth inhibition and apoptosis.

\section{Cross-resistance in CEM/ara-G cells}

The XTT assay also revealed that CEM/ara-G cells were cross-resistant to similar nucleoside analogs, ara- $C$ and fludarabine nucleoside F-ara-A (Table 1). Intracellular analog triphosphate production was also determined. CEM/ara-G cells yielded lower amounts of both ara-CTP and F-ara-ATP than CEM cells (Figure 3). Ara-CTP and F-ara-ATP were $3,400 \pm 400 \mathrm{pmol} / 10^{7}$ cells and $190 \pm 36 \mathrm{pmol} / 10^{7}$ cells in CEM cells, and $363 \pm 84$ $\mathrm{pmol} / 10^{7}$ cells and $29 \pm 13 \mathrm{pmol} / 10^{7}$ cells in CEM/ara-G 


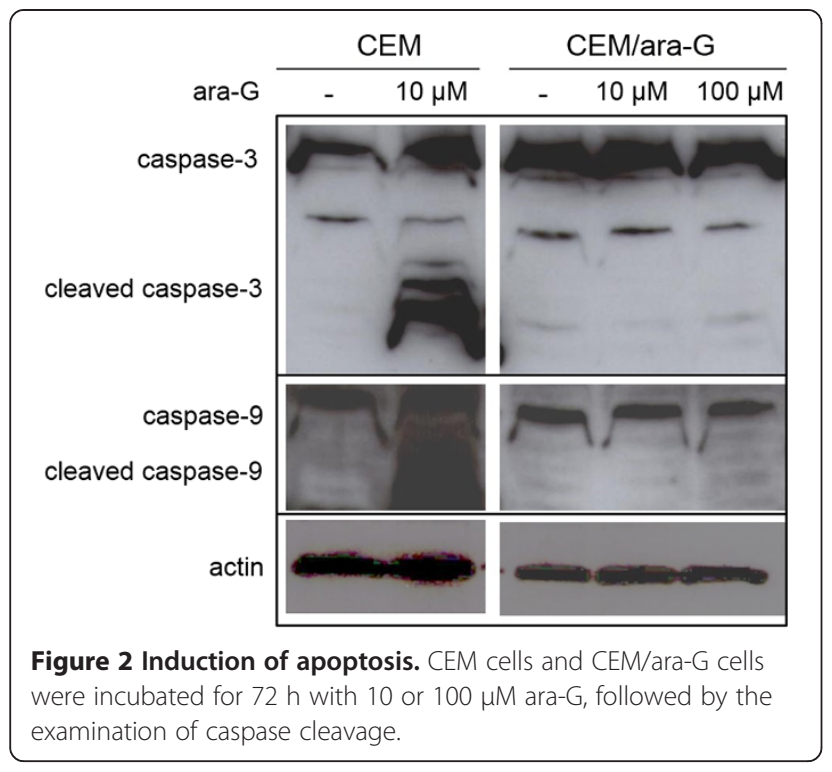

cells, respectively. Thus, the cross-resistance to ara- $\mathrm{C}$ and F-ara-A in CEM/ara-G cells was associated with the decreased production of intracellular analog triphosphates.

\section{Evaluation of factors (hENT1, dCK, dGK, and cN-II) essential for intracellular ara-GTP production}

The mechanism of resistance to nucleoside analogs is usually associated with impaired production of intracellular analog triphosphate [20,21]. The level of hENT1 transcript was decreased in CEM/ara-G cells (Figure 4a), suggesting a decreased cellular uptake of the nucleoside analog. Both $\mathrm{dCK}$ and $\mathrm{dGK}$ protein expression was also decreased in CEM/ara-G cells (Figure 4b). Transcript levels of the degrading enzyme cN-II were comparable between CEM cells and CEM/ara-G cells (Figure 4c). Thus, the cellular uptake and intracellular phosphorylation of ara-G were impaired in CEM/ara-G cells, which led to decreased araGTP production.

\section{Inhibition of DNA synthesis by the incorporation of ara-G} into DNA

The critical cytotoxic event of a nucleoside analog is incorporation of the intracellular analog triphosphate into nuclear DNA, thereby terminating DNA synthesis $[16,22,23]$. The uptake of thymidine into DNA was evaluated in the presence or absence of ara-G in both cell lines. Pre-incubation with $10 \mu \mathrm{M}$ ara-G, which is a concentration that is cytotoxic to CEM cells but not to CEM/ara-G cells, inhibited the incorporation of tritiated thymidine into both the nuclear and mitochondrial DNA fractions in CEM cells (Figure 5a, b). However, thymidine incorporation into DNA was not inhibited in either fraction of CEM/ ara-G cells (Figure 5a, b). Along with DNA synthesis inhibition, ara-G incorporation into DNA was evaluated in the nuclear and mitochondrial fractions of both cell lines. After treatment with $10 \mu \mathrm{M}$ ara-G, the amounts of ara-G incorporated into the DNA of both fractions of CEM/ara-G cells were reduced compared with those of CEM cells (Figure 5c). The reduction was comparable between the nuclear DNA and mitochondrial DNA fractions of CEM/ara-G cells (Figure 5c). The reduced incorporation of ara-G might correspond to the failed inhibition of thymidine incorporation (Figure 5a, b). Thus, CEM/ara-G cells were refractory to ara-G-mediated DNA synthesis inhibition of both nuclear and mitochondrial DNA fractions due to the reduced ara-G incorporation into DNA. The reduced ara-G incorporation might be attributable to the decreased production of intracellular ara-GTP in CEM/ ara-G cells.

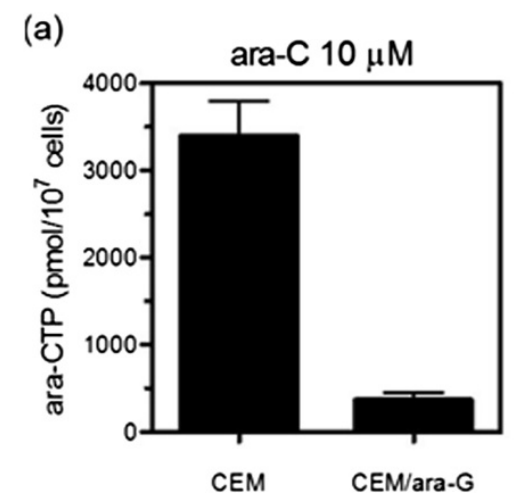

(b)

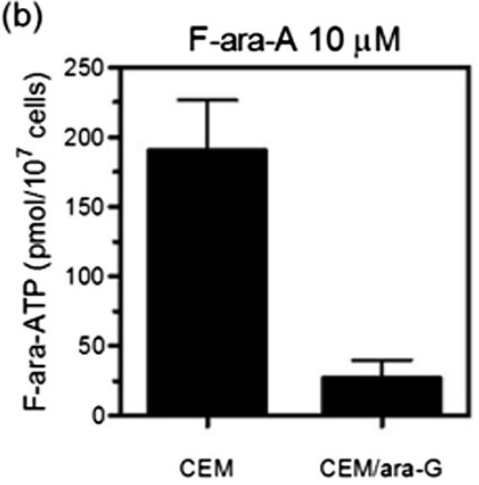

Figure 3 Intracellular analog triphosphate production. CEM cells and CEM/ara-G cells were incubated for 6 h with $10 \mu \mathrm{M}$ ara-C (a) or F-ara-A (b), followed by extraction of the nucleotide pool and measurement of intracellular analog triphosphate concentrations by using HPLC. P $=0.026$ for CEM versus CEM/ara-G for ara-CTP production by unpaired $T$ test (a). $P=0.001$ for CEM versus CEM/ara-G for F-ara-ATP production by unpaired $T$ test (b). The values shown are the means \pm SD of at least three independent experiments. 


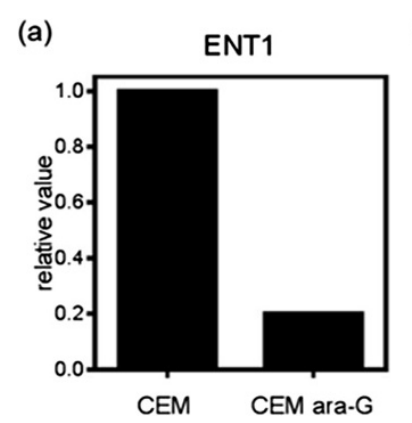

(b)

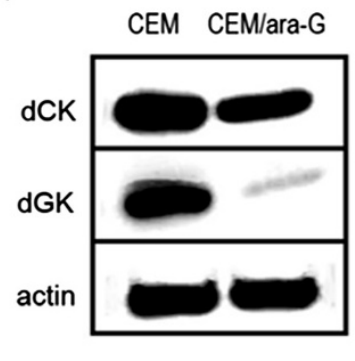

(c)

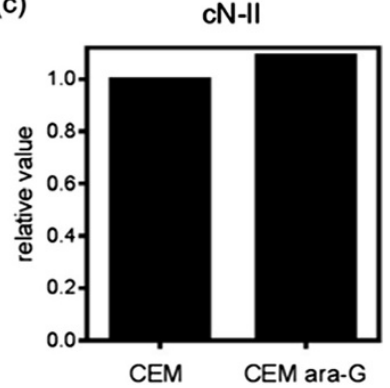

Figure 4 Factors associated with the intracellular activation of ara-G in CEM cells and CEM/ara-G cells. (a) Real-time RT-PCR was performed to determine the transcript level of hENT1. (b) Western blot analysis of dCK and dGK. (c) Real-time RT-PCR was performed to determine the transcript level of $\mathrm{CN}-\mathrm{Il}$.

\section{Derivation of mitochondrial DNA-depleted cells ( $\rho^{0}$ CEM cells)}

The role of the mitochondrial DNA damage in ara-G cytotoxicity was further evaluated. If mitochondrial DNA is a target of ara-G cytotoxicity, it was hypothesized that mitochondrial DNA-depleted cells would become resistant to ara-G. CEM cells were cultured in the presence of ethidium bromide to generate a mitochondrial DNA-depleted derivative $\left(\rho^{0} \mathrm{CEM}\right)$. The oxidase activity of cytochrome $c$, which is formed from subunits encoded by mitochondrial DNA, was almost absent in $\rho^{0}$ CEM cells (Figure 6a), indicating the successful depletion of mitochondrial DNA. The ATP-based proliferation assay revealed that the $\mathrm{IC}_{50}$ values were comparable between CEM cells and $\rho^{0}$ CEM cells (Table 2). The induction of apoptotic cell death was also evaluated in these cell lines. Intact mitochondrial function is not essential for inducing apoptosis because most $\rho^{0}$ cell lines undergo apoptosis in response to death signals and cytotoxic agents as efficiently as their parental cell lines
[24-27]. Ara-G induced apoptosis equally in CEM cells and $\rho^{0} \mathrm{CEM}$ cells, regardless of the ara-G concentration (Figure 6b, c). These results suggested that ara-G-induced mitochondrial DNA damage was unlikely to greatly contribute to ara-G cytotoxicity.

\section{Apoptosis-related proteins}

Apoptosis- and survival-related proteins were compared between CEM cells and CEM/ara-G cells (Figure 7). Antiapoptotic Bcl-xL was augmented and pro-apoptotic Bax and Bad were reduced in CEM/ara-G cells, suggesting refractoriness to ara-G-induced apoptosis. The levels of mitochondrial apoptosis-related proteins, including Bcl-2, $\mathrm{Bcl}-\mathrm{xL}, \mathrm{Bax}, \mathrm{Bad}, \mathrm{Bid}$, and Bim, were not altered in $\rho^{0} \mathrm{CEM}$ cells. Pro-survival AKT and P-AKT levels were equivalent among CEM cells, CEM/ara-G cells, and $\rho^{0}$ CEM cells [28].

\section{Discussion}

In the present study, we developed a new cell line variant of the $\mathrm{T}$ lymphoblastic leukemia CCRF-CEM cell
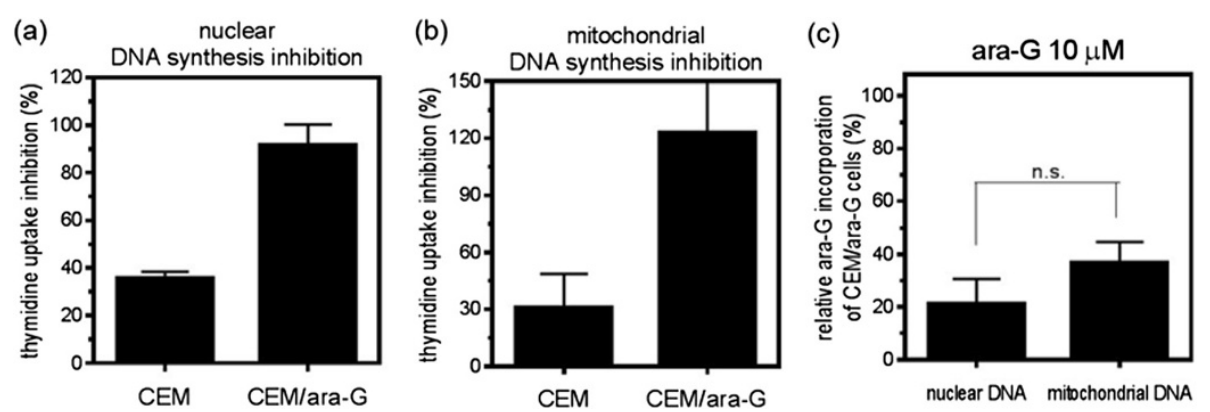

Figure 5 DNA synthesis inhibition by ara-G. CEM cells and CEM/ara-G cells were incubated with or without $10 \mu \mathrm{M}$ ara-G for 3 h, followed by a 4-h incubation with tritiated thymidine. Nuclear (a) and mitochondrial (b) DNA fractions were isolated and subjected to scintillation counting. Percentages are the ratio of the values of thymidine incorporation into the DNA of the cells that had been pre-treated with ara-G relative to those without ara-G pre-incubation. $P=0.0003$ for CEM versus CEM/ara-G for nuclear DNA synthesis inhibition by unpaired $T$ test. $P=0.045$ for CEM versus CEM/ara-G for mitochondrial DNA synthesis inhibition by unpaired $T$ test. (c) CEM and CEM/ara-G cells were incubated with $10 \mu M$ radio-labeled ara-G for $6 \mathrm{~h}$, followed by extraction of nuclear and mitochondrial DNA. Then, the samples were subjected to scintillation counting. The relative ara-G incorporation is the ratio of the value of ara-G incorporation into the DNA of CEM/ara-G cells to that of CEM cells. n.S., not significant. The values shown are the means \pm SD of at least three independent experiments. 


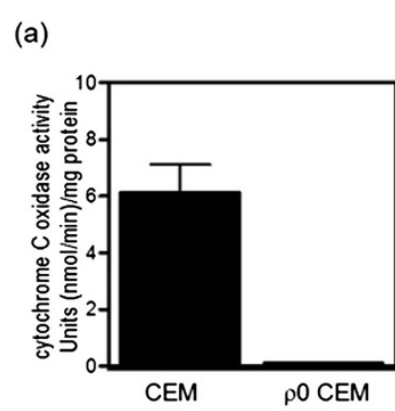

(b)

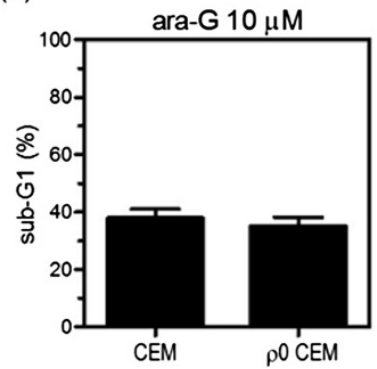

(c)

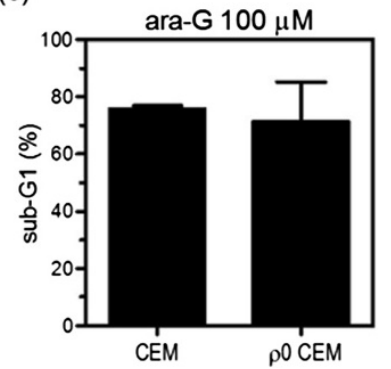

Figure 6 Ara-G cytotoxicity against mitochondrial DNA-depleted CEM ( $\left.\rho^{\circ} \mathrm{CEM}\right)$ cells. (a) Determination of cytochrome c oxidase activity in $\rho^{0} \mathrm{CEM}$ cells. The activity was completely suppressed in mitochondrial DNA-depleted variant cell line $\rho^{0} \mathrm{CEM}$ as compared with CEM cells. (b, c) CEM cells and $\rho^{\circ} \mathrm{CEM}$ cells were treated with $10 \mu \mathrm{M}$ (b) or $100 \mu \mathrm{M}$ (c) ara-G for $48 \mathrm{~h}$. Sub-G1 induction was calculated by using flow cytometry. The values shown are the means \pm SD of at least three independent experiments. The difference in the values between CEM cells and $\rho^{\circ} \mathrm{CEM}$ cells was not significant for either concentration ( $P=0.28$ for $10 \mu \mathrm{M}$ ara-G (b), $P=0.40$ for $100 \mu \mathrm{M}$ ara-G (c), unpaired $T$ test).

line, which was resistant to ara-G, an active compound of nelarabine (Figures 1 and 2, Table 1), and investigated its mechanism of drug resistance. Reduced transporter hENT1 transcript level and decreased dCK and dGK protein levels (Figure 4) resulted in decreased araGTP production (Figure 1) in CEM/ara-G cells. The subsequent incorporation of ara-G into nuclear and mitochondrial DNA was reduced (Figure 5), and unable to inhibit DNA synthesis in both fractions of CEM/ ara-G cells (Figure 5). Importantly, the cytotoxic effect of ara-G was almost unchanged on CEM cells that were depleted of mitochondrial DNA (Figure 6, Table 2), suggesting that mitochondrial DNA damage was unlikely to contribute greatly to ara-G cytotoxicity. Thus, the reduced triphosphate production (Figure 1) and the subsequent reduction of drug incorporation into nuclear DNA (Figure 5) were closely associated with the development of ara-G resistance in CEM/ara-G cells. The antiapoptotic nature was also related to the drug resistance in this cell line (Figure 7).

Previously, 3 independent studies investigated the mechanisms of ara-G resistance in leukemic cell lines. Shewach et al. first developed an ara-G-resistant leukemic clone from $\mathrm{T}$ lymphoblastic leukemia MOLT-4 cells and demonstrated decreased production of intracellular araGTP [29]. However, they did not determine the mechanisms for the reduced ara-GTP production. Curbo et al. generated 2 ara-G-resistant CEM subclones that were

Table 2 Drug sensitivity of CEM cells after the loss of mitochondrial DNA

\begin{tabular}{lcc}
\hline & \multicolumn{2}{c}{$\mathrm{IC}_{50}(\mu \mathrm{M})$} \\
\cline { 2 - 3 } Drug & $\mathrm{CEM}$ & $\boldsymbol{\rho}^{\mathbf{0}} \mathrm{CEM}$ \\
\hline Ara-G & 3.5 & 4.0 \\
\hline $\begin{array}{l}\text { CEM cells and mitochondria-depleted } \rho^{\mathbf{0}} \mathrm{CEM} \text { cells were incubated for } 72 \mathrm{~h} \\
\text { with various concentrations of ara-G. The IC } \\
\text { the ATP-based assay. }\end{array}$
\end{tabular}

132-fold and 260-fold more ara-G resistant than CEM [30]. They demonstrated a decrease in ara-G incorporation into mitochondrial DNA and loss of dCK activity. However, they showed that the drug incorporation into mitochondrial DNA was not associated with the acute

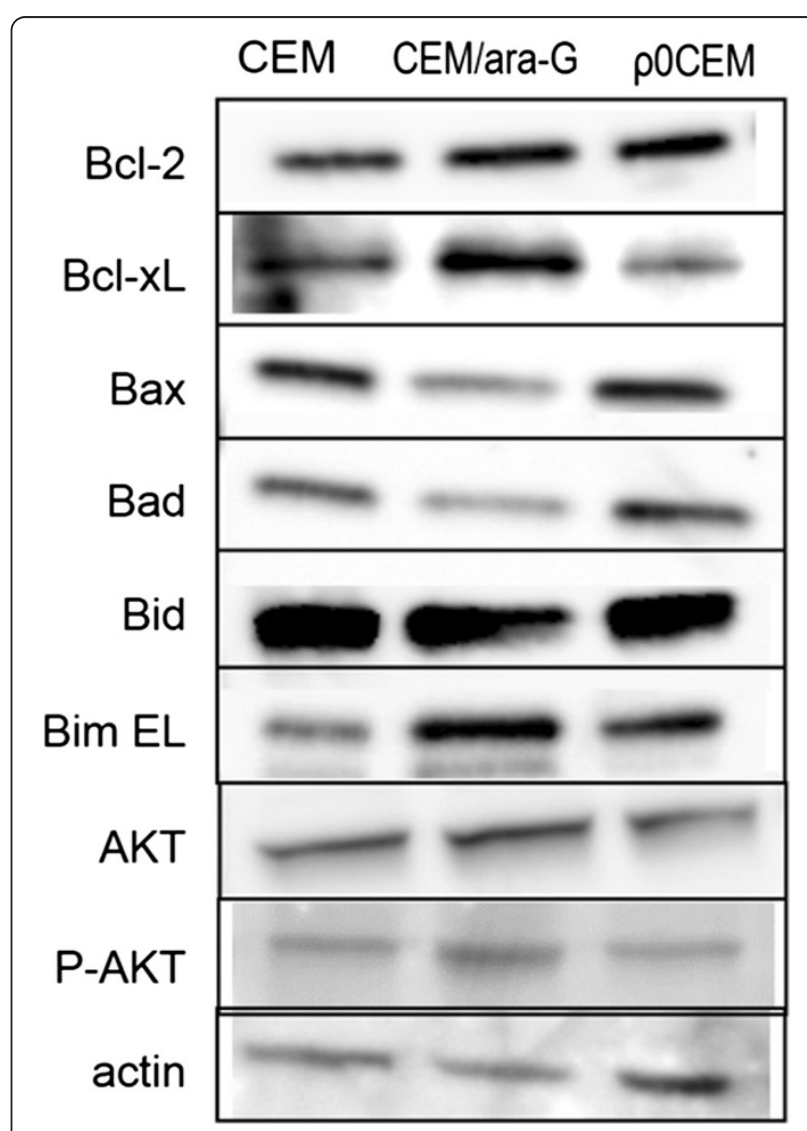

Figure 7 Protein levels of $\mathrm{Bcl}-2, \mathrm{Bcl}-\mathrm{xL}, \mathrm{Bax}, \mathrm{Bad}, \mathrm{Bid}, \mathrm{Bim} \mathrm{EL}$ (extra long), AKT, and phospho-AKT. These levels were determined by Western blotting in CEM cells, CEM/ara-G cells, and $\rho^{0} \mathrm{CEM}$ cells. 
cytotoxicity induced by ara-G in their later study [31]. Their latest study further demonstrated that the depletion of mitochondria DNA does not attenuate the cytotoxicity of ara-G in MOLT-4 cells [32]. They concluded that the loss of dCK activity is the critical factor responsible for ara-G resistance. Our study demonstrated that ara-G inhibited both nuclear and mitochondrial DNA synthesis in CEM cells (Figure 5). However, the result showing that $\rho^{0} \mathrm{CEM}$ cells were similarly sensitive to ara-G (Figure 6) suggests that the critical event should be the inhibition of nuclear DNA synthesis not mitochondrial DNA damage. Lotfi et al. developed 2 ara-G-resistant MOLT-4 variants that were 108-fold and 184-fold more ara-G resistant than MOLT-4 [33]. They showed that dGK deficiency was the most prominent change in these cells and that a dCK defect was associated with increased ara-G resistance [33]. They further identified increases in Bcl-xL in these ara-Gresistant clones [34]. The alteration of the kinases and anti-apoptotic $\mathrm{Bcl}-\mathrm{xL}$ indicate a possible contribution of these factors to ara-G resistance, which is consistent with our present findings. Nevertheless, apart from these reports, we clearly showed all of the successive changes in the transporter hENT1, kinases (dCK and dGK), ara-GTP production, ara-G incorporation into nuclear and mitochondrial DNA, inhibition of DNA synthesis, and induction of mitochondria-mediated apoptosis. Thus, unlike previous studies, the present study was comprehensive and systematic in investigating the mechanism of resistance to ara-G in leukemic cells.

CEM/ara-G cells demonstrated cross-resistance to $\mathrm{F}$-ara-A and ara-C. However, the resistance to the purine analog $\mathrm{F}$-ara-A was much greater than that to the pyrimidine analog ara-C (Table 1). Because F-ara-A and ara-C share an identical pathway for their intracellular activation, the difference in resistance might be due to a structural difference between the 2 agents, but this possibility was not investigated in detail here. Nevertheless, one strategy to overcome ara- $\mathrm{G}$ resistance may be a high-dose ara- $\mathrm{C}$ therapy that can achieve 50-fold higher plasma ara- $\mathrm{C}$ concentrations than regular-dose ara- $C$, which would surpass the level of cross-resistance to ara- $C[35,36]$.

\section{Conclusions}

An ara-G-resistant CEM variant was successfully established. The mechanism of resistance included reduced drug incorporation into nuclear DNA and antiapoptosis.

\footnotetext{
Abbreviations

ara-G: 9- $\beta-D$-arabinofuranosylguanine; ara-GTP: 9- $\beta$-D-arabinofuranosylguanine triphosphate; F-ara-A: 9- $\beta$-D-arabinofucanosyl-2-fluoroadenine; F-ara-ATP: 9- $\beta$-Darabinofucanosyl-2-fluoroadenine triphosphate; ara-C: Cytarabine; araCTP: Cytarabine triphosphate; XTT: Sodium 3'-(1-[(phenylamino)-carbonyl3,4-tetrazolium])-bis(4-methoxy-6-nitro)benzene sulfonic acid hydrate; hENT1: Human Equilibrative Nucleoside Transporter 1; dCK: Deoxycytidine kinase; dGK: Deoxyguanosine kinase; CN-II: Cytosolic 5'-nucleotidase II; $\mathrm{IC}_{50}$ : 50\% growth-inhibitory concentration.
}

\section{Competing interests}

The authors have nothing to disclose concerning any of the drugs or agents used in the present study.

\section{Authors' contributions}

TY conceived the design of the study and performed the data analysis. KU carried out growth inhibition analysis and Western blotting. RN carried out HPLC analysis. HS carried out Western blotting. TU participated in its design and coordination and helped to draft the manuscript. All authors read and approved the final manuscript.

\section{Acknowledgments}

This work was supported in part by grants from the Gout Research Foundation $(2008,2009,2010)$. The role of the funding body was in design, in the collection, analysis, and interpretation of data, and in the writing and the submission of the manuscript.

Received: 11 February 2014 Accepted: 23 July 2014

Published: 29 July 2014

\section{References}

1. Faderl S, Gandhi V, Kantarjian H, Plunkett W: New nucleoside analogues in clinical development. Cancer Chemother Biol Response Modif 2002, 20:37-58.

2. Liu X, Kantarjian H, Plunkett W: Sapacitabine for cancer. Expert Opin Investig Drugs 2012, 21:541-555.

3. Ghanem $\mathrm{H}$, Kantarjian $\mathrm{H}$, Ohanian M, Jabbour $\mathrm{E}$ : The role of clofarabine in acute myeloid leukemia. Leuk Lymphoma 2013, 54:688-698.

4. Cohen MH, Johnson JR, Massie T, Sridhara R, McGuinn WD Jr, Abraham S, Booth BP, Goheer MA, Morse D, Chen XH, Chidambaram N, Kenna L, Gobburu JV, Justice R, Pazdur R: Approval summary: nelarabine for the treatment of T-cell lymphoblastic leukemia/lymphoma. Clin Cancer Res 2006, 12:5329-5335.

5. Gandhi V, Plunkett W: Clofarabine and nelarabine: two new purine nucleoside analogs. Curr Opin Oncol 2006, 18:584-590.

6. Ravandi F, Gandhi V: Novel purine nucleoside analogues for T-cell-lineage acute lymphoblastic leukaemia and lymphoma. Expert Opin Investig Drugs 2006, 15:1601-1613.

7. DeAngelo DJ, Yu D, Johnson JL, Coutre SE, Stone RM, Stopeck AT, Gockerman JP, Mitchell BS, Appelbaum FR, Larson RA: Nelarabine induces complete remissions in adults with relapsed or refractory T-lineage acute lymphoblastic leukemia or lymphoblastic lymphoma: cancer and leukemia group B study 19801. Blood 2007, 109:5136-5142.

8. Rodriguez CO Jr, Gandhi V: Arabinosylguanine-induced apoptosis of T-lymphoblastic cells: incorporation into DNA is a necessary step. Cancer Res 1999, 59:4937-4943.

9. Rodriguez CO Jr, Mitchell BS, Ayres M, Eriksson S, Gandhi V: Arabinosylguanine is phosphorylated by both cytoplasmic deoxycytidine kinase and mitochondrial deoxyguanosine kinase. Cancer Res 2002, 62:3100-3105.

10. Rodriguez CO Jr, Stellrecht CM, Gandhi V: Mechanisms for T-cell selective cytotoxicity of arabinosylguanine. Blood 2003, 102:1842-1848.

11. Gandhi V, Mineishi S, Huang P, Yang Y, Chubb S, Chapman AJ, Nowak BJ, Hertel LW, Plunkett W: Difluorodeoxyguanosine: cytotoxicity, metabolism, and actions on DNA synthesis in human leukemia cells. Semin Oncol 1995, 22(4 Suppl 11):61-67.

12. Prus KL, Averett DR, Zimmerman TP: Transport and metabolism of 9- $\beta-D-$ arabinofuranosylguanine in a human T-lymphoblastoid cell line: nitrobenzylthioinosine-sensitive and -insensitive influx. Cancer Res 1990, 50:1817-1821.

13. Shigemi H, Yamauchi T, Tanaka Y, Yokota Y, Ueda T: Establishment of novel leukemic cell lines resistant to clofarabine by dual mechanisms of decreased intracellular active metabolite and increased antiapoptotic factor. Cancer Sci 2013, 104:732-739.

14. Yamauchi T, Nishi R, Kitazumi K, Nakano T, Ueda T: A new highperformance liquid chromatography method determines low production of 9-beta-D-arabinofuranosylguanine triphosphate, an active metabolite of nelarabine, in adult T-cell leukemia cells. Oncol Rep 2010, 23:499-504 
15. Yamauchi T, Negoro E, Kishi S, Takagi K, Yoshida A, Urasaki Y, Iwasaki H, Ueda T: Intracellular cytarabine triphosphate production correlates to deoxycytidine kinase/cytosolic 5'-nucleotidase II expression ratio in primary acute myeloid leukemia cells. Biochem Pharmacol 2009, 77:1780-1786.

16. Yamauchi T, Ueda T: A sensitive new method for clinically monitoring cytarabine concentrations at the DNA level in leukemic cells. Biochem Pharmacol 2005, 69:1795-1803.

17. Yamauchi T, Nowak BJ, Keating MJ, Plunkett W: DNA repair initiated in chronic lymphocytic leukemia lymphocytes by 4-hydroperoxycyclophosphamide is inhibited by fludarabine and clofarabine. Clin Cancer Res 2001, 7:3580-3589.

18. Yamauchi T, Keating MJ, Plunkett W: UCN-01 (7-hydroxystaurosporine) inhibits DNA repair and increases cytotoxicity in normal lymphocytes and chronic lymphocytic leukemia lymphocytes. Mol Cancer Ther 2002, 1:287-294.

19. Park KS, Nam KJ, Kim JW, Lee YB, Han CY, Jeong JK, Lee HK, Pak YK: Depletion of mitochondrial DNA alters glucose metabolism in SK-Hep1 cells. Am J Physiol Endocrinol Metab 2001, 280:E1007-E1014.

20. Yamauchi T, Ueda T, Nakamura T: A new sensitive method for determination of intracellular 1- $\beta$-D-arabinofuranosylcytosine 5 '-triphosphate content in human materials in vivo. Cancer Res 1996, 56:1800-1804.

21. Yamamoto S, Yamauchi T, Kawai Y, Takemura H, Kishi S, Yoshida A, Urasaki Y, Iwasaki H, Ueda T: Fludarabine-mediated circumvention of cytarabine resistance is associated with fludarabine triphosphate accumulation in cytarabine-resistant leukemic cells. Int J Hematol 2007, 85:108-115.

22. Huang P, Plunkett W: Fludarabine- and gemcitabine- induced apoptosis: incorporation of analogs into DNA is a critical event. Cancer Chemother Pharmacol 1995, 36:181-188.

23. Huang P, Robertson LE, Wright S, Plunkett W: High molecular weight DNA fragmentation: a critical event in nucleoside analogue-induced apoptosis in leukemia cells. Clin Cancer Res 1995, 1:1005-1013.

24. Jacobson MD, Burne JF, King MP, Miyashita T, Reed JC, Raff MC: BCl-2 blocks apoptosis in cells lacking mitochondrial DNA. Nature 1993, 361:365-369.

25. Marchetti P, Susin SA, Decaudin D, Gamen S, Castedo M, Hirsch T, Zamzami N, Naval J, Senik A, Kroemer G: Apoptosis-associated derangement of mitochondrial function in cells lacking mitochondrial DNA. Cancer Res 1996, 56:2033-2038.

26. Liang $B C$, Ullyatt $E$ : Increased sensitivity to cis-diamminedichloroplatinum induced apoptosis with mitochondrial DNA depletion. Cell Death Differ 1998, 5:694-701.

27. Jiang S, Cai J, Wallace DC, Jones DP: Cytochrome c-mediated apoptosis in cells lacking mitochondrial DNA. Signaling pathway involving release and caspase 3 activation is conserved. J Biol Chem 1999, 274:29905-29911.

28. Pelicano H, Xu RH, Du M, Feng L, Sasaki R, Carew JS, Hu Y, Ramdas L, Hu L, Keating MJ, Zhang W, Plunkett W, Huang P: Mitochondrial respiration defects in cancer cells cause activation of Akt survival pathway through a redox-mediated mechanism. J Cell Biol 2006, 175:913-923.

29. Shewach DS, Daddona PE, Ashcraft E, Mitchell BS: Metabolism and selective cytotoxicity of 9-beta-D-arabinofuranosylguanine in human lymphoblasts. Cancer Res 1985, 45:1008-1014.

30. Curbo S, Zhu C, Johansson M, Balzarini J, Karlsson A: Dual mechanisms of 9- $\beta$-D-arabinofuranosylguanine resistance in CEM T-Lymphoblast Leukemia cells. Biochem Biophys Res Com 2001, 285:40-45.

31. Curbo S, Zhivotovsky B, Johansson M, Karlsson A: Effects of 9- $\beta-D-$ arabinofuranosylguanine on mitochondria in CEM T-lymphoblast leukemia cells. Biochem Biophys Res Com 2003, 307:942-947.

32. Curbo S, Johansson M, Balzarini J, Lewis LD, Karlsson A: Acute cytotoxicity of arabinofuranosyl nucleoside analogs is not dependent on mitochondrial DNA. Exp Cell Res 2009, 315:2539-2543.

33. Lotfi K, Månsson E, Peterson C, Eriksson S, Albertioni F: Low level of mitochondrial deoxyguanosine kinase is the dominant factor in acquired resistance to 9- $\beta$-D-arabinofuranosylguanine cytotoxicity. Biochem Biophys Res Com 2002, 293:1489-1496.

34. Månsson E, Stridh H, Albertioni F: Resistance to mitochondrial- and Fas-mediated apoptosis in human leukemic cells with acquired resistance to 9- $\beta$-D-arabinofuranosylguanosine. Biochem Biophys Res Com 2002, 298:338-344.

35. Plunkett W, lacoboni S, Estey E, Danhauser L, Liliemark JO, Keating MJ: Pharmacologically directed ara-C therapy for refractory leukemia. Semin Oncol 1985, 12(suppl. 3):20-30.

36. Estey EH: Treatment of relapsed and refractory acute myelogenous leukemia. Leukemia 2000, 14:476-479.

doi:10.1186/1471-2407-14-547

Cite this article as: Yamauchi et al:: Reduced drug incorporation into DNA and antiapoptosis as the crucial mechanisms of resistance in a novel nelarabine-resistant cell line. BMC Cancer 2014 14:547.

\section{Submit your next manuscript to BioMed Central and take full advantage of:}

- Convenient online submission

- Thorough peer review

- No space constraints or color figure charges

- Immediate publication on acceptance

- Inclusion in PubMed, CAS, Scopus and Google Scholar

- Research which is freely available for redistribution

Submit your manuscript at www.biomedcentral.com/submit
C BioMed Central 